\title{
Synthesis of CdSe/ZnS and CdTe/ZnS Quantum Dots: Refined Digestive Ripening
}

\author{
Sreeram Cingarapu, ${ }^{1}$ Zhiqiang Yang, ${ }^{2}$ Christopher M. Sorensen, ${ }^{3}$ and Kenneth J. Klabunde ${ }^{1}$ \\ ${ }^{1}$ Department of Chemistry, Kansas State University, Manhattan, KS 66506, USA \\ ${ }^{2}$ Department of Chemistry, Clemson University, Clemson, SC 29634, USA \\ ${ }^{3}$ Department of Physics, Kansas State University, Manhattan, KS 66506, USA
}

Correspondence should be addressed to Kenneth J. Klabunde, kenjk@ksu.edu

Received 27 September 2011; Accepted 4 November 2011

Academic Editor: Bo Zou

Copyright (c) 2012 Sreeram Cingarapu et al. This is an open access article distributed under the Creative Commons Attribution License, which permits unrestricted use, distribution, and reproduction in any medium, provided the original work is properly cited.

We report synthesis of CdSe and CdTe quantum dots (QDs) from the bulk CdSe and CdTe material by evaporation/cocondensation using the solvated metal atom dispersion (SMAD) technique and refined digestive ripening. The outcomes of this new process are (1) the reduction of digestive ripening time by employing ligands (trioctylphosphine oxide (TOPO) and oleylamine $(\mathrm{OA})$ ) as capping agent as well as digestive ripening solvent, (2) ability to tune the photoluminescence (PL) from $410 \mathrm{~nm}$ to $670 \mathrm{~nm}$, (3) demonstrate the ability of SMAD synthesis technique for other semiconductors (CdTe), (4) direct comparison of CdSe QDs growth with CdTe QDs growth based on digestive ripening times, and (5) enhanced PL quantum yield (QY) of CdSe QDs and CdTe QDs upon covering with a ZnS shell. Further, the merit of this synthesis is the use of bulk $\mathrm{CdSe}$ and CdTe as the starting materials, which avoids usage of toxic organometallic compounds, eliminates the hot injection procedure, and size selective precipitation processes. It also allows the possibility of scale up. These QDs were characterized by UV-vis, photoluminescence (PL), transmission electron microscopy (TEM), high-resolution transmission electron microscopy (HRTEM), X-ray photoelectron spectroscopy (XPS), and powder XRD.

\section{Introduction}

The solvated metal atom dispersion (SMAD) technique allows the synthesis of nanomaterial from the bulk material by vaporization and cocondensation [1-8]. The as-prepared poly-dispersed SMAD colloid products were made monodispersed in size by a unique process known as digestive ripening [9]. Digestive ripening involves the heating of polydispersed colloidal material at or near the boiling point (BP) of solvent in the presence of excess surface active ligand [1-11]. In the present work, we employed trioctylphosphine oxide (TOPO) and oleylamine (OA), which served both as capping agent as well as digestive ripening solvent. The general procedure for the synthesis of high-quality crystalline II-VI semiconductor material is by the hot injection method, where cadmium precursor $\left(\mathrm{CH}_{3}\right)_{2} \mathrm{Cd}$ or $\mathrm{CdO}$ is dissolved in coordination ligands like trioctylphosphine oxide, hexylphosphonic acid, or tetradecylphosphonic acids, and then the selenium precursor (Se dissolved in TOP) quickly injected into the hot coordination reaction mixture, which initiated the nucleation process, and subsequent growth was carried out at a relatively lower temperature, and this process was initially reported by Murray et al. [12], and later, Peng et al. and Talapin et al. have developed the hot injection procedure [13-19]. One of the advancements in this process was selecting an injection temperature and a growth temperature. This high reaction temperature $(>150$ $350^{\circ} \mathrm{C}$ ) facilitates the removal of crystalline defects and allows enhancement in the photoluminescence. In semiconductor QDs, high emission efficiency from a band-edge state is required especially when these are used in lasers or imaging. In general, a high band gap inorganic material coating over the QD core has been proven to enhance the QY by passivating surface nonradiative recombination sites. Typically, II-VI semiconductor QDs are covered with a high band gap ZnS shell, which was initially developed 


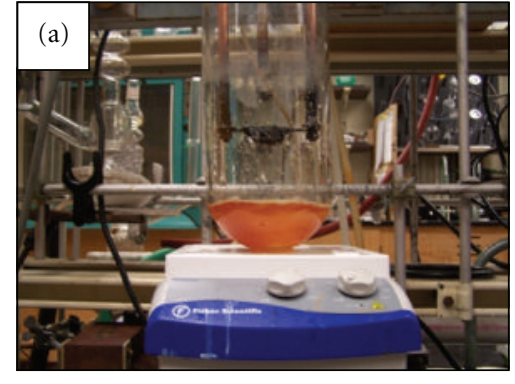

(a)

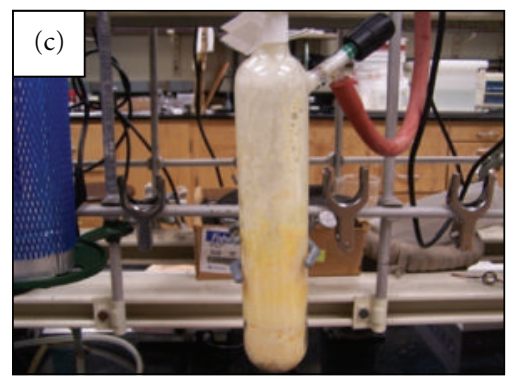

(c)

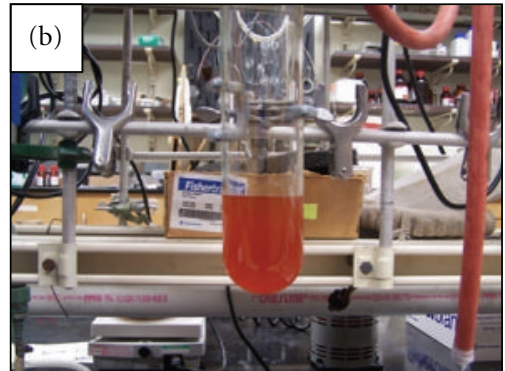

(b)

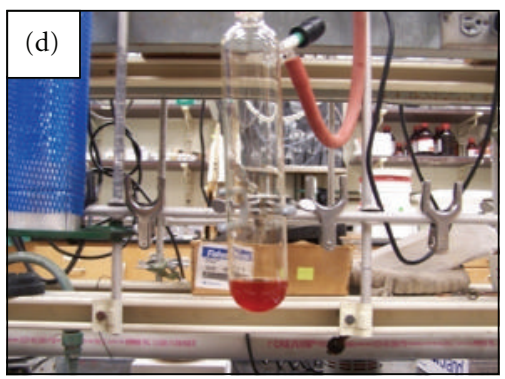

(d)

FIGURE 1: (a) as-prepared SMAD CdSe-THF-TOPO-OA colloidal solution after vigorous stirring for a period of 45 minutes (b) as-prepared siphoned CdSe-THF-TOPO-OA colloidal solution (c) semi-solid CdSe-TOPO-OA after complete vacuum evaporation of THF solvent (d) CdSe-TOPO-OA colloid after gentle warming.

by Hines and Guyot-Sionnest [20]. These two methods (hot injection and $\mathrm{ZnS}$ shell covering) have been widely used to achieve narrow-size particle distribution and enhance QY. In addition to the above method, other routes like layer by layer $\mathrm{ZnS}$ passivation [21], $\mathrm{CO}_{2}$ gas-expanded liquids [22], surface treatments with polymers [23], and sonochemical processes [24] were used. In the current work, we adopted the sonochemical procedure for the growth of $\mathrm{ZnS}$ shell over CdSe and CdTe QD core.

\section{Experimental Section}

2.1. Chemicals. Bulk cadmium selenide(CdSe) and bulk cadmium telluride (CdTe) (99.9\%, Strem Chemicals Inc), oleylamine (98\%) from ACROS Organic chemicals, trioctylphosphine oxide (TOPO) (Reagent Plus 99\%), trioctylphosphine (TOP), tributyl phosphine $\left(\mathrm{PBu}_{3}\right)$, zinc nitrate hexahydrate, and potassium ethylxanthate were purchased from SigmaAldrich and used without further purification. Tetrahydrofuran (THF), acetone, and methanol were purchased from Fisher Scientific. Tetrahydrofuran solvents were distilled and degassed four times by the standard freeze-thaw procedure prior to use. Oleyl amine was purged with argon for $2 \mathrm{hrs}$ prior to use.

2.2. Synthesis of as-Prepared SMAD Colloid. A stationary reactor [25] was used for the evaporation and cocondensation of bulk CdSe or CdTe. Briefly, $1 \mathrm{~g}$ of either bulk CdSe or bulk CdTe was evaporated using water cooled copper electrodes and the generated heat during the evaporation was dissipated by water cooled copper electrodes and insulating packing material (Zircar product, Inc.) around the crucible and metal basket. The optimum temperature required for the evaporation of bulk CdSe is $\sim 900^{\circ} \mathrm{C}$, whereas, for bulk $\mathrm{CdTe}$, it is less than $900^{\circ} \mathrm{C}$. Initially the bulk material was charged in C9 boron nitride crucible (R.D. Mathis \# C9-BN) resting in a metal basket (R. D. Mathis \# B8B \# X.030 w), and the ligands were placed at the bottom of the SMAD reactor and the entire setup was then vacuum sealed. After complete evacuation, a liquid $\mathrm{N}_{2}$ Dewar was placed around the sealed SMAD reactor. Once the vacuum attains $4 \times 10^{-3}$ torr, initially $50 \mathrm{~mL}$ of distilled and degassed THF was evaporated through a solvent shower head, which was inserted into the reactor. The evaporated solvent was condensed on the wall of SMAD reactor by external liquid nitrogen cooling. After the formation of condensed solvent matrix on the walls, the metal crucible was heated by water cooled copper electrode, and the heat was ramped slowly and the evaporated material was cocondensed along with the solvent on to the walls of reactor. Cocondensation of evaporate material along with the solvent restricts aggregation and allows formation of small crystallites. It took nearly $3 \mathrm{hrs}$ for the complete evaporation of $1 \mathrm{~g}$ of bulk material. The frozen matrix appears reddish brown (Figure 1). Upon warming up of the frozen matrix with a heat gun, the matrix melts and slowly reaches the bottom of the reactor and mixes well with the coordinating ligands (TOPO with OA). To ensure homogeneous colloid formation, the system was vigorously stirred for 45 minutes with a magnetic stirrer. Figure 1(a) shows the as-prepared CdSe-THF-TOPO-OA colloidal solution after vigorous stirring. The as-prepared SMAD product was then siphoned 


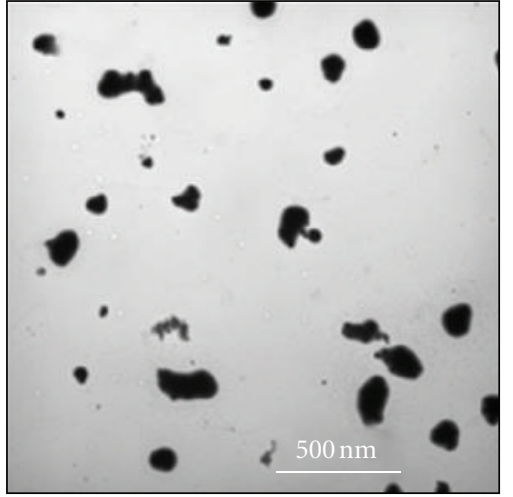

(a)

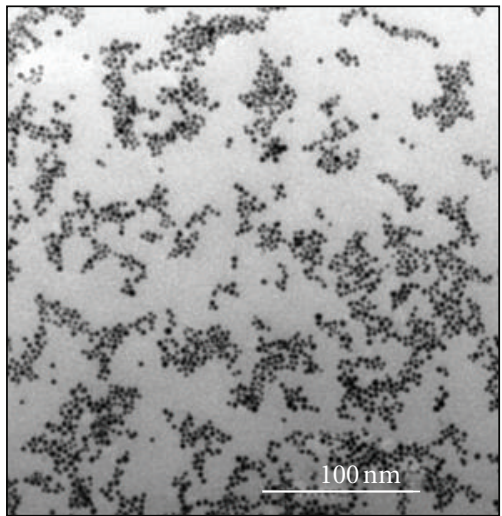

(d)

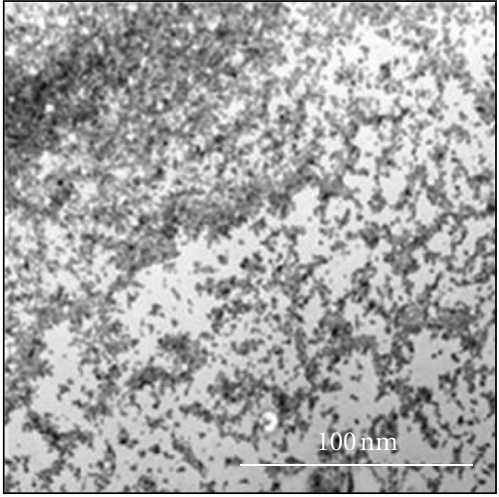

(b)

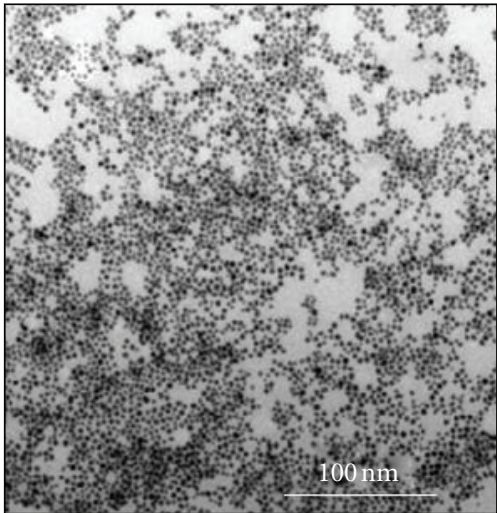

(e)

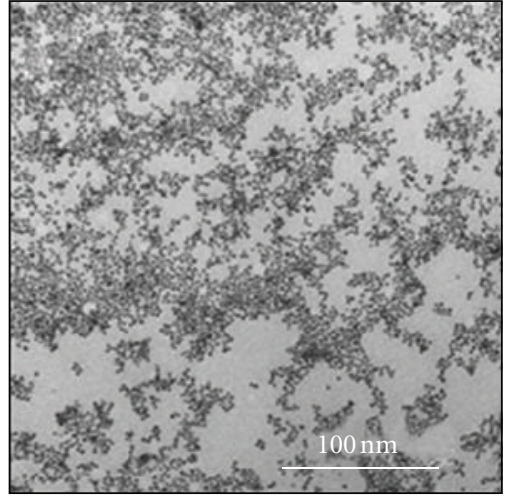

(c)

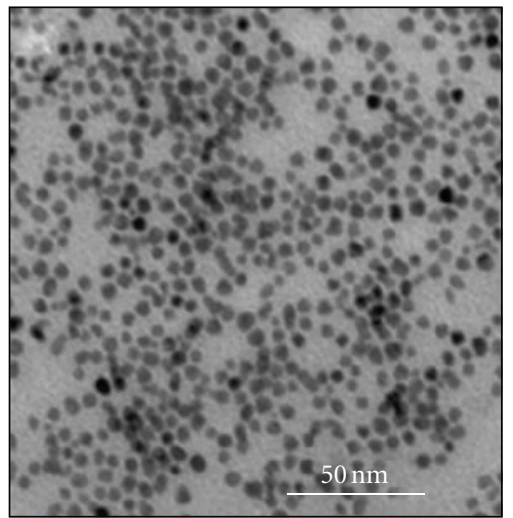

(f)

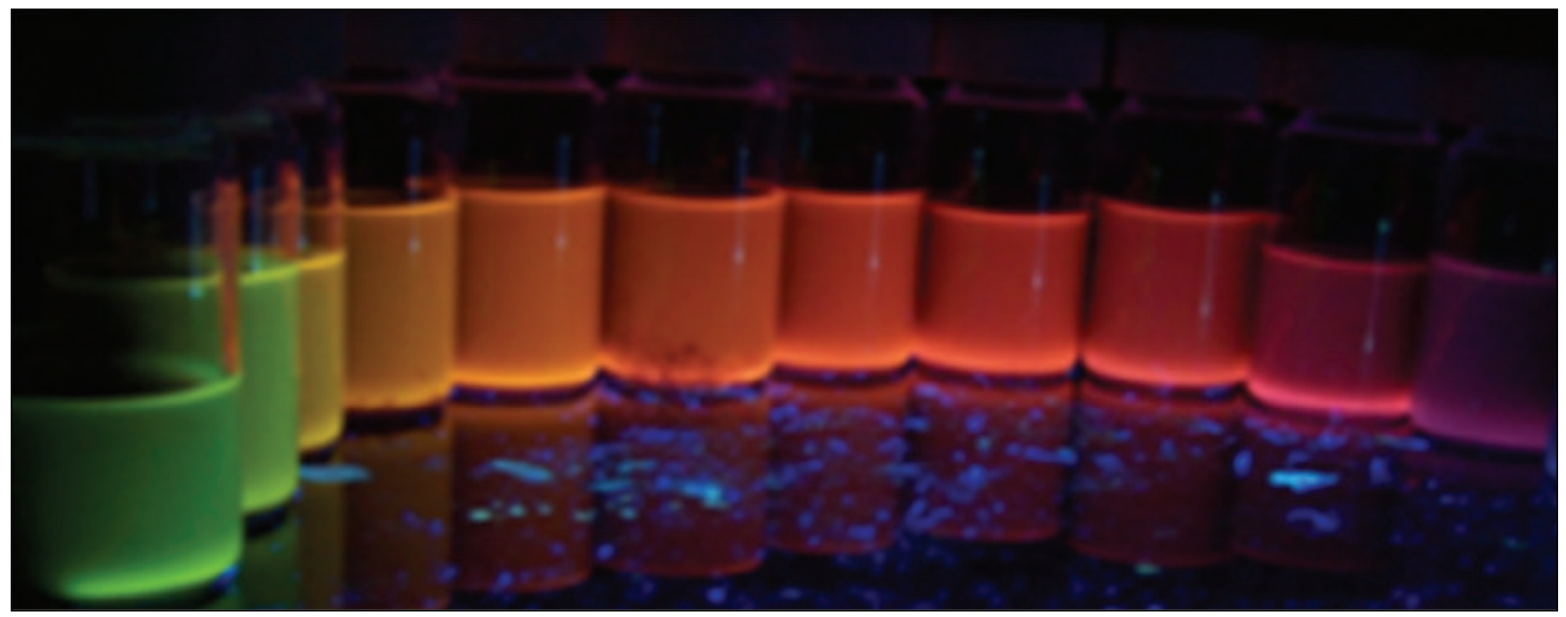

(g)

Figure 2: The TEM images of (a) as-prepared SMAD product, (b) after $10 \mathrm{~min}$ (c) after 20 min and (d) after 40 min of digestive ripening. (e) after $60 \mathrm{~min}$, and (f) after $90 \mathrm{~min}$ of digestive ripening (g) CdSe QDS samples collected at various intervals of digestive ripening time upon exposure to UV-Vis illuminator.

into a Schlenk glass tube under the protection of argon (Figure 1(b)). Safety and cleanliness: prior to synthesis, the SMAD reactor was cleaned with aqua regia, base bath, acid bath, and finally with copious amount of water. While working with vacuum lines it's a must to wear protective eye glasses. Both CdSe and CdTe are carcinogenic so, proper protection is necessary while handing these chemicals. Also, the acid and base bath used in cleaning may cause severe burns, so proper acid proof gloves and protecting clothing are necessary.

2.3. Preparation of CdSe-TOPO-OA Colloid. The THF from the as-prepared CdSe-THF-TOPO-OA colloidal solution was vacuum evaporated, leaving a THF solvent free semisolid CdSe-TOPO-OA colloid (Figure 1(c)). Upon gentle warming, CdSe-TOPO-OA colloid was obtained (Figure 1(d)). 


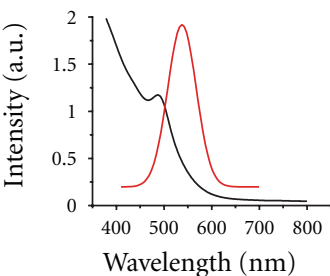

(a)

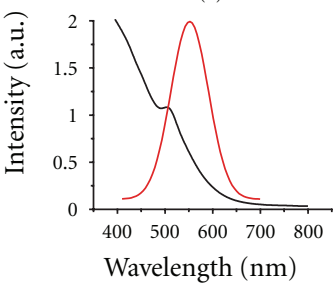

(e)

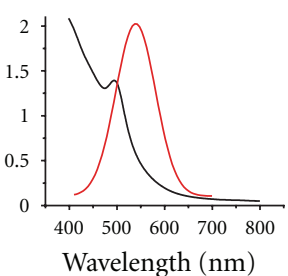

(b)

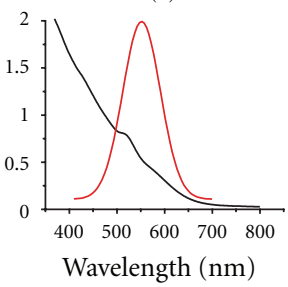

(f)

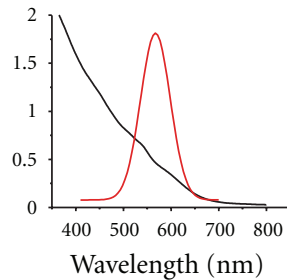

(c)

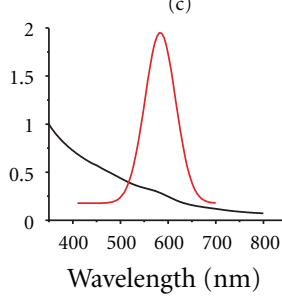

(g)

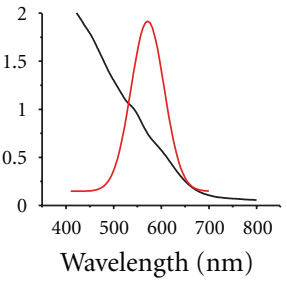

(d)

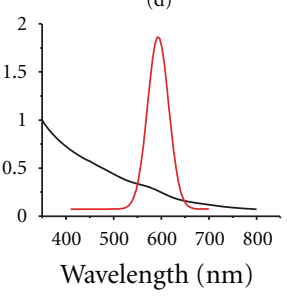

(h)

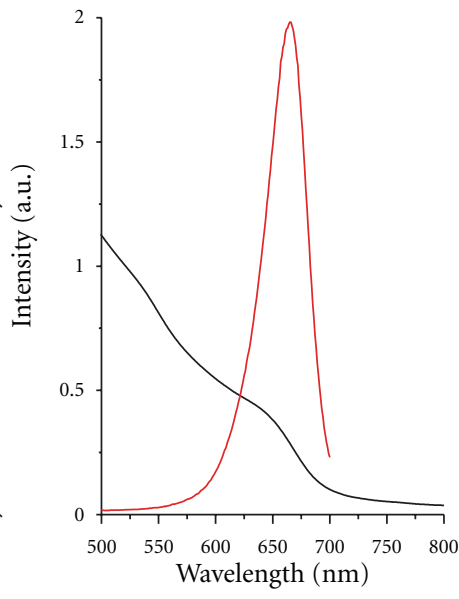

(i)

FIGURe 3: (a)-(i) UV-vis absorption spectra and corresponding PL of CdSe QD samples collected at 10, 20, 30, 40, 50, 60, 70, 80, and 90 minutes of digestive ripening.

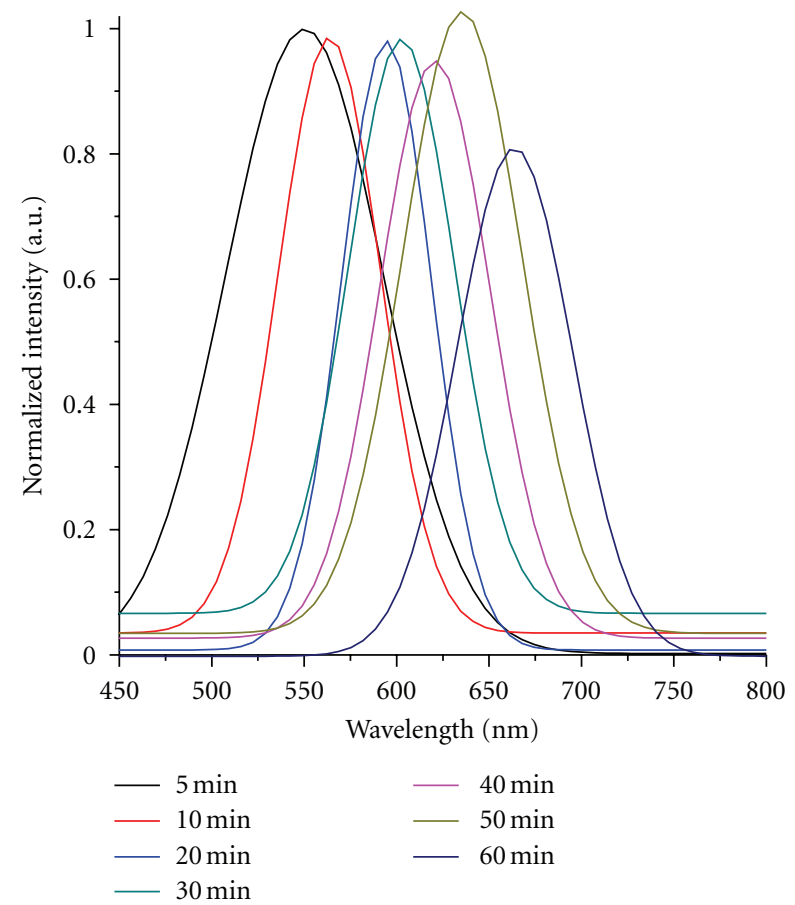

FIGURE 4: Photoluminescence spectrum of CdTe QD samples collected at 5, 10, 20, 30, 40, 50, and 60 minutes of digestive ripening.

This as-prepared product was then subjected to digestive ripening. The same procedure was adopted for the synthesis of the as-prepared CdTe-TOPO-OA system.

2.4. Refined Digestive Ripening. Digestive ripening is the key step for the formation of quasimonodispersed core QDs. In previous work, we used toluene and t-butyl toluene as a digestive ripening solvent for the CdSe-TOPO-HDA system. However, for the current work TOPO and OA were used as digestive ripening solvent, as well as capping ligands.
2.5. ZnS Shell Formation on Core Quantum Dots. ZnS shell formation over a core QD (CdSe or CdTe) was carried out by a reported sonochemical procedure using zinc ethylxanthate as precursor $[24,26]$. In a typical $\mathrm{ZnS}$ shell growth, aliquots $(5 \mathrm{~mL})$ of freshly synthesized core QDs were placed in a reaction vessel and then it was placed in $100 \mathrm{~W}$ sonicator (Fisher Scientifics), to which freshly prepared zinc ethylxanthate $(0.15 \mathrm{~g})$ in tributylphosphine $(3 \mathrm{~mL})\left(\mathrm{PBu}_{3}\right)$ solution was mixed when the sonication temperature was $60^{\circ} \mathrm{C}$. The sonication was continued until the temperature of the reaction mixture reached to $120^{\circ} \mathrm{C}$ to ensure complete passivation of the QD core with the ZnS shell. During this process, aliquots of reaction mixture were collected to monitor the shell growth and no purification steps were involved on the core solution before use. Isolation of coreshell QDs was carried out by precipitation with anhydrous methanol, followed by washing with acetone and methanol. This process was repeated to remove any unreacted zinc ethylxanthate and excess ligands. The coreshell QDs were then vacuum dried and redispersed in toluene for transmission electron microscope (TEM) sample preparation. No size selective precipitation step was carried out. The yield of core QDs is about $\sim 78-80 \%$.

\section{Characterization}

3.1. UV-vis Spectroscopy. UV-vis absorption spectra were obtained using an in situ UV-vis optical fiber, assisted by a DH-2000 UV-vis optical spectrophotometer instrument (Ocean Optics Inc) for core QDs. The absorption spectra of core-shell QDs were obtained using a Cary 500 Scan UV-visNIR spectrophotometer. All samples were washed with absolute ethanol, acetone, and were dried under vacuum. The dried samples were then redissolved in toluene for analysis.

3.2. Photoluminescence Spectroscopy. Fluorescence spectra of both core QDs (CdSe and CdTe) and core-shell QDs (CdSe$\mathrm{ZnS}$ and $\mathrm{CdTe}-\mathrm{ZnS}$ ) were measured by using a Fluoro Max-2 


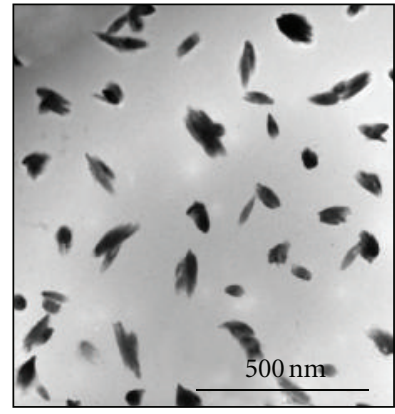

(a)

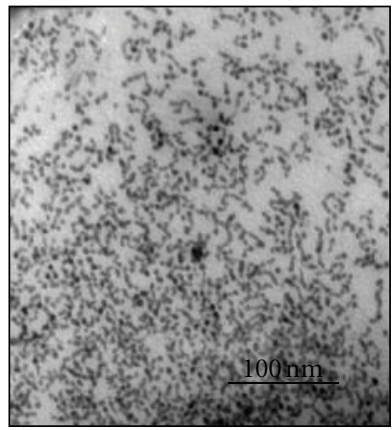

(e)

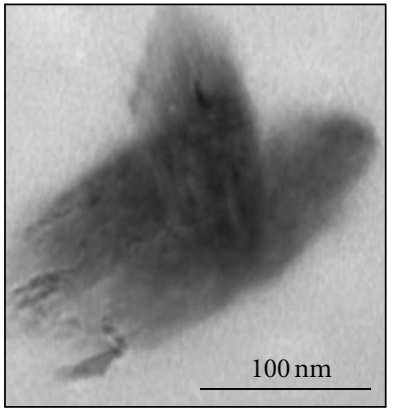

(b)

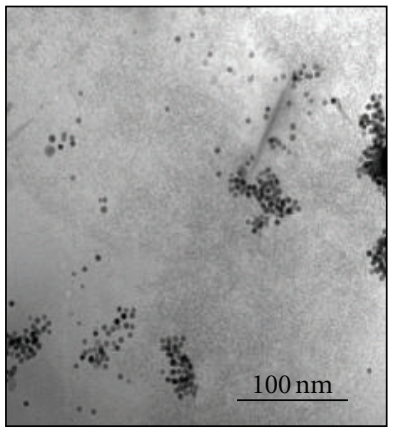

(f)

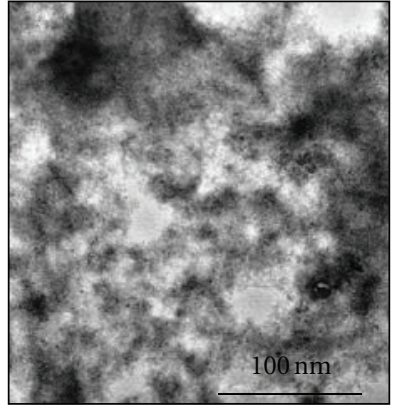

(c)

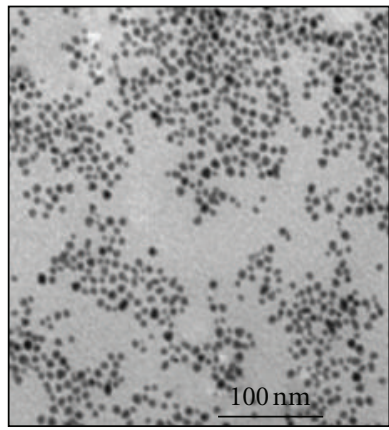

(g)

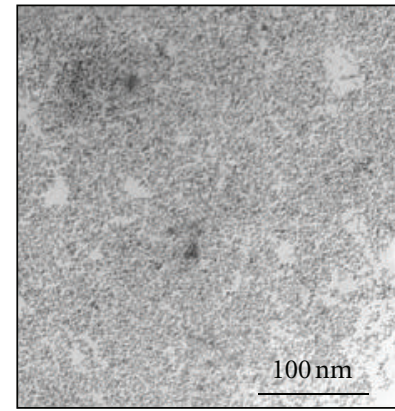

(d)

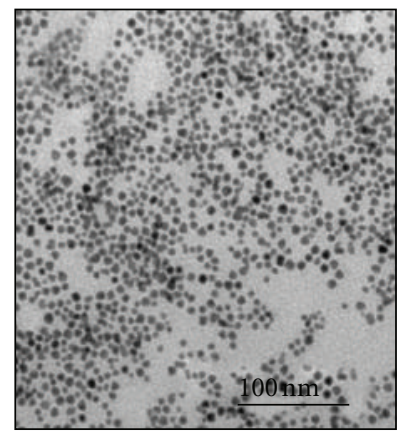

(h)

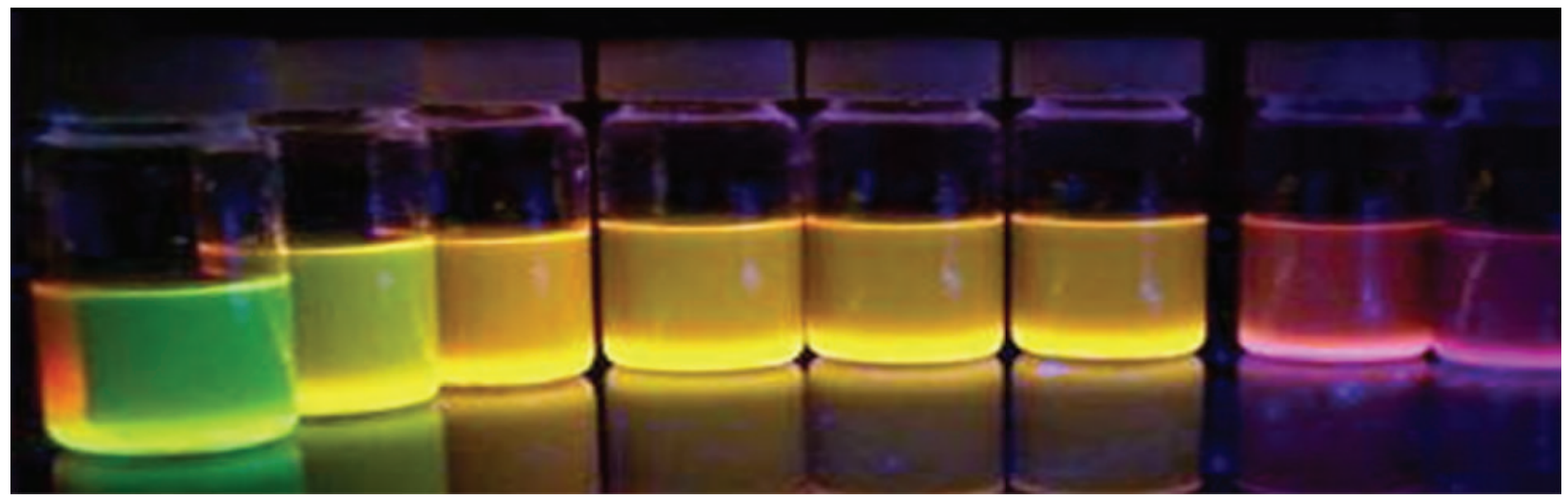

(i)

FIGURE 5: TEM images of CdTe QDs, (a, b) as-prepared SMAD product before digestive ripening, (c) after 10 min of digestive ripening, (d) after $20 \mathrm{~min}$, (e) after $30 \mathrm{~min}$ (f) after $40 \mathrm{~min}$ of digestive ripening, (g) after $50 \mathrm{~min}$ and (h) after 60 min of digestive ripening, (i) samples collected at various intervals of digestive ripening time under UV-vis illuminator.

instrument from HORIBA Jobin Yvon Company. These samples were all excited at $400 \mathrm{~nm}$. Photoluminescence quantum yields (QY) value (Фem) of QDs (CdSe and CdTe) and coreshell QDs (CdSe-ZnS and CdTe-ZnS) were measured relative to Rhodamine 6G in methanol, assuming it's PL QYs as $95 \%$ $[27,28]$, and the $\%$ yield were calculated by using $(1)$.

$$
\Phi e m=\Phi S\left(\frac{\mathrm{I}}{\mathrm{IS}}\right)\left(\frac{\mathrm{AS}}{\mathrm{A}}\right)\left(\frac{\mathrm{n} 2}{\mathrm{nS} 2}\right)
$$

In 1, I (sample) and IS (standard) are the integrated emission peak areas, up to $480 \mathrm{~nm}$ excitation. A (sample) and AS (standard) are the absorption $(<0.1)$ at $480 \mathrm{~nm}$; n (sample) and $\mathrm{nS}$ (standard) are the refractive indices of the solvents; and the Фem and $\Phi S$ are the PL QYs for the sample and the standard, respectively.

3.3. Transmission Electron Microscopy (TEM). TEM Studies were Performed on a Philips CM100 operating at $100 \mathrm{kV}$. The TEM Samples were Prepared by placing a few micro liters of precipitated, washed, vacuum dried and redissolved sample in toluene onto a carbon-coated formvar copper grid and the grids were allowed to dry overnight.

\subsection{High Resolution Transmission Electron Microscopy/Energy} Dispersive X-Ray Spectroscopy. High-resolution images were performed with a FEI Tecnai F20 XT field emission transmission electron microscopy operated at $300 \mathrm{kV}$. The energy 


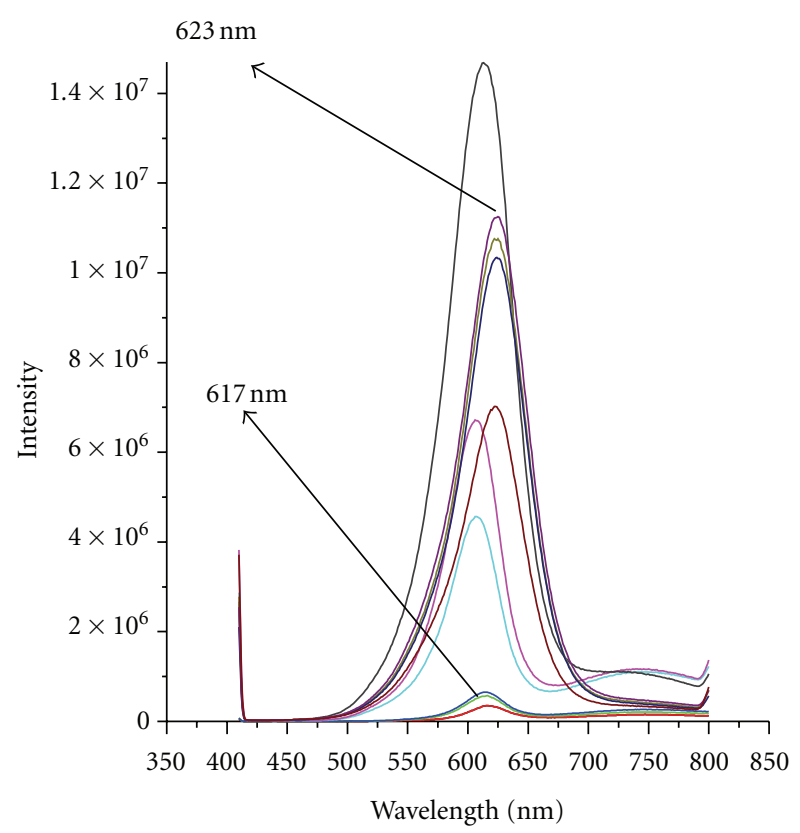

- CdSe core after 10 min sonication

- CdSe core after 20 min sonication CdSe core after $30 \mathrm{~min}$ sonication

- CdSe/ZnS after 5 min of sonication

— CdSe/ZnS after $10 \mathrm{~min}$ of sonication

- CdSe/ZnS after $15 \mathrm{~min}$ of sonication

- CdSe/ZnS after $20 \mathrm{~min}$ of sonication

- CdSe/ZnS after $25 \mathrm{~min}$ of sonication

- CdSe/ZnS after $30 \mathrm{~min}$ of sonication

- CdSe/ZnS after $35 \mathrm{~min}$ of sonication

— CdSe/ZnS after $40 \mathrm{~min}$ of sonication

FIGURE 6: Shell coating on digestively ripened CdSe QDs. The broad emission band from $625-700 \mathrm{~nm}$ wavelength in core CdSe QDs is due to surface traps. But upon $\mathrm{ZnS}$ shell growth, the emission due to surface traps was reduced and simultaneously enhanced PL can be seen. The PL shift from $540 \mathrm{~nm}$ to $545 \mathrm{~nm}$ and then to $555 \mathrm{~nm}$ indicates the growth of shell material on the CdSe Core.

dispersive X-ray (EDX) analysis was carried out in a scanning electron microscope (SEM) mode, which is an integral part of FEI Tecnai F20 XT. The experimental conditions are as follow: energy resolution: $134 \mathrm{eV}$, reference energy: $5.9 \mathrm{keV}$, minimum energy: $100 \mathrm{eV}$, detector thickness: $3 \mathrm{~mm}$, detector distance: $11.8 \mathrm{~mm}$, and detector angle: $14.6^{\circ}$. The facilities were provided by the Microscopy and Analytical Imaging Laboratory at the University of Kansas. Washing procedures were the same as described above. However, a Lacey-carboncoated TEM grid was used.

3.5. Powder X-Ray Diffraction (PXRD). Powder X-ray diffraction patterns were recorded by a Bruker D8 X-ray diffractometer with $\mathrm{CuK} \alpha$ radiation. PXRD samples were prepared by the evaporation of toluene from the core or coreshell QDs/toluene dispersion loaded on XRD glass plates. The samples were scanned from $20<2 \theta<80^{\circ}$ at an increment of $0.01^{\circ} / \mathrm{min}$, and the total acquisition time period was more than $7 \mathrm{hrs}$.
3.6. X-Ray Photoelectron Spectroscopy. XPS data were collected on a Kratos Axis $165 \mathrm{X}$-ray photoelectron spectrometer operating in the hybrid mode using $\mathrm{Al} \mathrm{K} \alpha(1486.6 \mathrm{eV})$ radiation at $300 \mathrm{~W}$. Charge neutralization was used to minimize sample charging, and the charge neutralizer settings were 2.0 amps, $1.7 \mathrm{~V}$ charge balance, and $1.1 \mathrm{~V}$ bias. Survey spectra were collected with pass energy of $160 \mathrm{eV}$, while highresolution spectra were collected with a pass energy of $20 \mathrm{eV}$. Peak fitting was performed using CASA XPS software, using peaks with a 50\% Gaussian, and 50\% Lorentzian line shape on a Shirley background. The facilities were provided by the shared experimental facilities (SEF) at the University of Maryland.

\section{Results and Discussion}

4.1. Cadmium Selenide-Oleylamine-TrioctylPhosphine Oxide. The as-prepared SMAD product generally yields polydispersed colloid material due to little control over the particle size during vaporization and cocondensation [1, 2, 5-7]. In previous work [1], we reported synthesis of CdSe QD from bulk by vaporization/cocondensation, followed by digestive ripening in toluene for $24 \mathrm{hrs}$ and $16 \mathrm{hrs}$ in t-butyl toluene. In the current refined digestive ripening, we used TOPO and $\mathrm{OA}$ in 30:20 ratio to the bulk starting material, which act as both digestive ripening solvent as well as capping agent. The as-prepared CdSe-TOPO-OA-THF (Figure 1(a)) colloid material is a homogeneous single phase. In order to obtain CdSe-TOPO-OA colloid, the THF solvent was vacuum evaporated resulting a semisolid CdSe-TOPO-OA (Figure 1(b)) and the semi-solid nature (Figure 1(c)) of the CdSe-TOPO-OA can be explained by the fact that TOPO exists as a solid at room temperature, whereas $\mathrm{OA}$ is liquid. The yield of CdSe QD is nearly $\sim 80 \%$ with variations in batch to batch synthesis and the $20 \%$ loss was due to either condensation of vaporized material onto copper electrodes, or possibly to losses in handling. In this work, we also employed OA as a solvent, as others have done [15, 29]. Compared to TOPO, OA is a weaker ligand and binds less strongly to the CdSe, and thereby affects the growth process $[27,30]$. The as-prepared CdSe exhibited a broad fluorescence, but within 90 minutes of digestive ripening, the PL becomes sharper and can be tuned to $670 \mathrm{~nm}$. The reduction of digestive ripening time is attributed to the higher boiling point of these ligands/Solvents. The poorfluorescence behavior of the as-prepared CdSe is due to aggregation of small crystallites, which is evident from the TEM image (Figure 2(a)).

During digestive ripening these aggregates of small crystallites (Figure 2(a)), breakdown completely to form small particles (Figure 2(b)) and grow into quasimonodispersed particles (Figure 2(b)-2(f)), Figure 2(g) is the samples (collected at various intervals of digestive ripening time) under UV-vis illuminator.

It is important to note that in digestive ripening bigger particles will break down into smaller particles and the smaller particles will tend to grow bigger and finally the system will reach a thermodynamic equilibrium size, and 


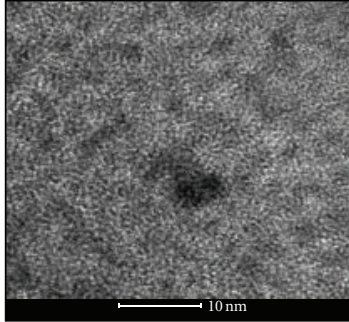

(a)

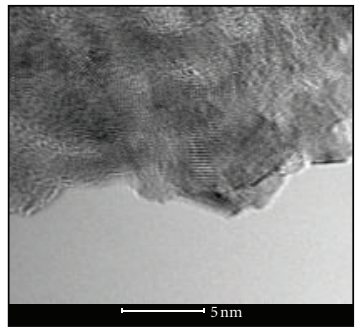

(c)

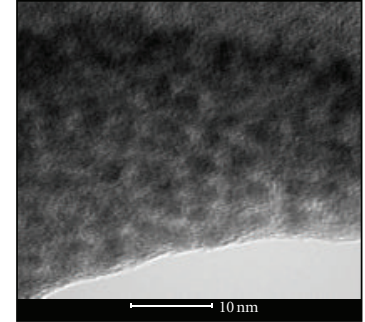

(b)

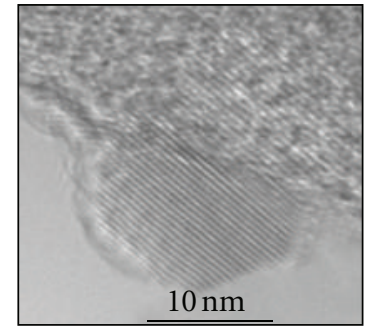

(d)

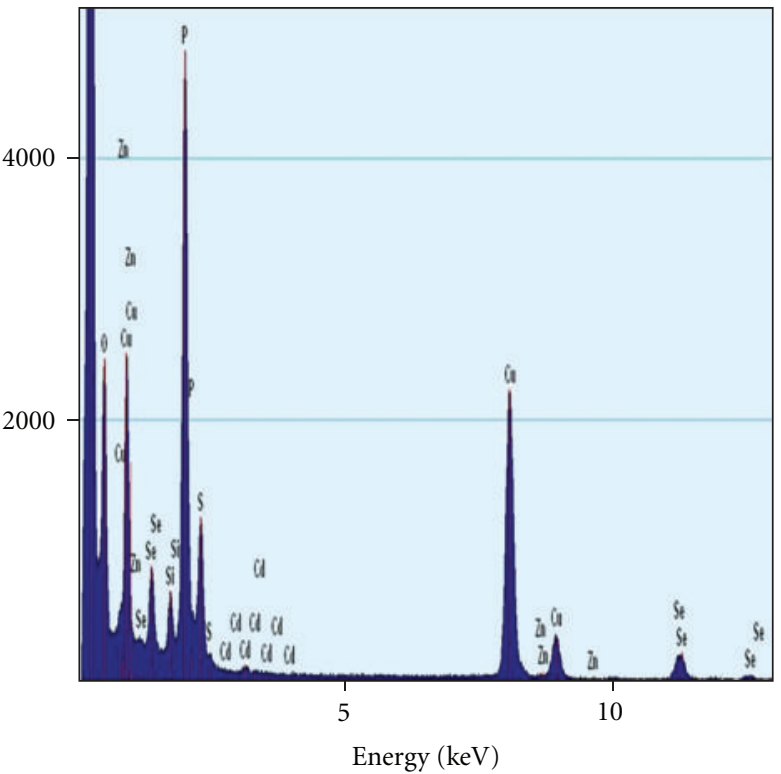

(e)

Figure 7: (a)-(b) HRTEM image of CdSe QD obtained after 30 and 90 minutes of digestive ripening, (c-d) CdSe/ZnS Core-Shell QDs showing the crystalline lattice and (e) energy dispersion spectroscopy (EDX) spectrum of CdSe- ZnS core-shell QDs showing characteristic peaks of cadmium, selenide, zinc and sulfur. Copper is from the lacy-carbon-coated copper TEM grid.

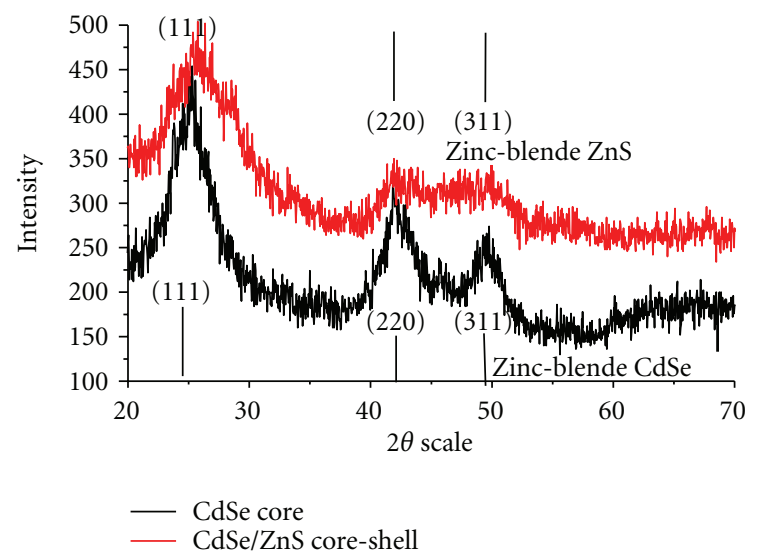

FIGURE 8: The XRD patterns of CdSe (black lines) showing the characteristic features of zinc blend crystalline structure and $\mathrm{CdSe} / \mathrm{ZnS}$ (red lines) showing the characteristic features of zinc blend structure, and the vertical bars (bottom) correspond to JCPDS file No. 77-2100 and (top) JCPDS file No. 19-0191.

the PL data of CdSe can be correlated with the digestive ripening phenomenon. With digestive ripening time, the UV-Vis spectra shifts to longer wavelength and the PL peaks sharpen (Figure 3(a)-3(i)) indicating the formation of quantum confined quasimonodispersed quantum dots. A more description about digestive ripening phenomenon can be found in latest review article and the reference therein [31].

4.2. CadmiumTelluride-Oleylamine-Trioctyl Phosphine Oxide. In case of CdTe, a similar trend was observed where; the as-prepared SMAD product has broad fluorescence peak but upon digestive ripening the fluorescence peak narrows and shifts in wavelength. Under similar $\left(250^{\circ} \mathrm{C}\right)$ digestive ripening temperature, the PL of CdTe can be tuned to $667 \mathrm{~nm}$ but compared to CdSe it took only 60 minutes. The as-prepared SMAD product is light yellow and turns brighter yellow color, then red and finally to dark red, which indicates rapid breakdown of aggregated crystallites and dramatic growth of CdTe nanocrystals in the first 30 minutes of digestive ripening. After 30 minutes, the growth rate decreased, indicating the formation of larger particles. Figure 4 shows the PL of CdTe QDs with the progress of digestive ripening time. As observed with $\mathrm{CdSe}$, CdTe also reaches an equilibrium size; where the initial FWHM was broad but as digestive ripening time progresses the FWHM has narrowed.

Figure 5(a) shows the as-prepared SMAD product TEM images. Note the aggregated small crystallites in Figure 5(b), but upon digestive ripening, these aggregates breakdown into smaller particles and then grow in size (Figure 5(c)-5(h)). Figure 5(i) shows the corresponding samples collected at various time intervals from the same batch and these samples were exposed to the UV-Vis illuminator.

4.3. Cadmium Selenide Core-Zinc Sulfide Shell QDs. Over coating of semiconductor QDs with a high band gap inorganic material enhances the photoluminescence QY by passivating surface recombination sites [20]. Surface coating not only protects the core nanomaterial from photooxidation but also from physical and chemical stress. The evolution of the PL from the CdSe- $\mathrm{ZnS}$ is depicted in Figure 6. It is clearly shown that the PL peak becomes sharp and the red 


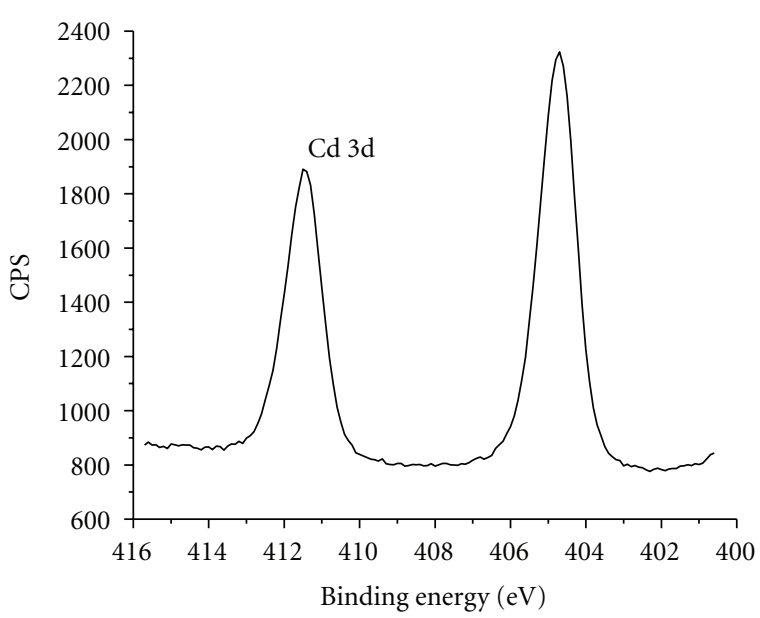

(a)

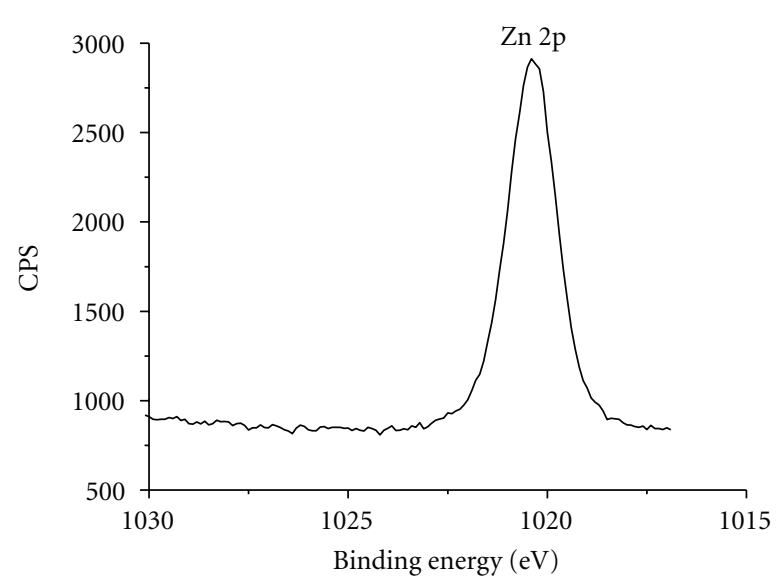

(c)

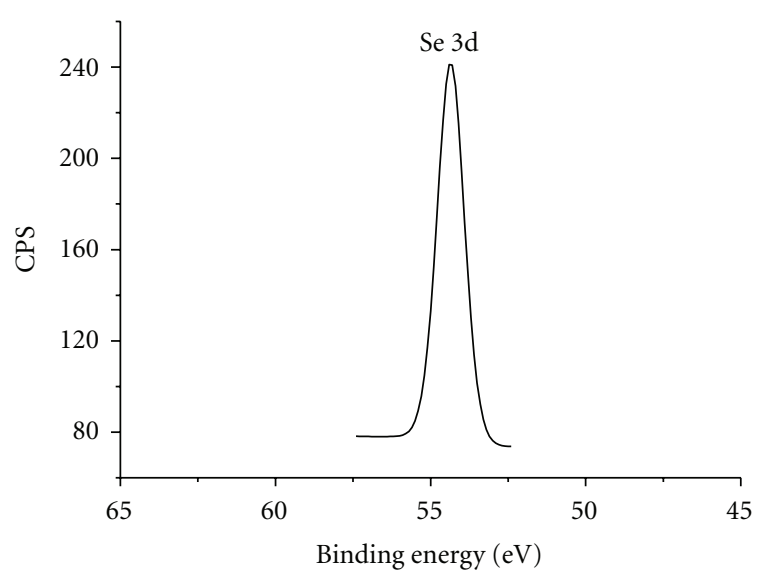

(b)

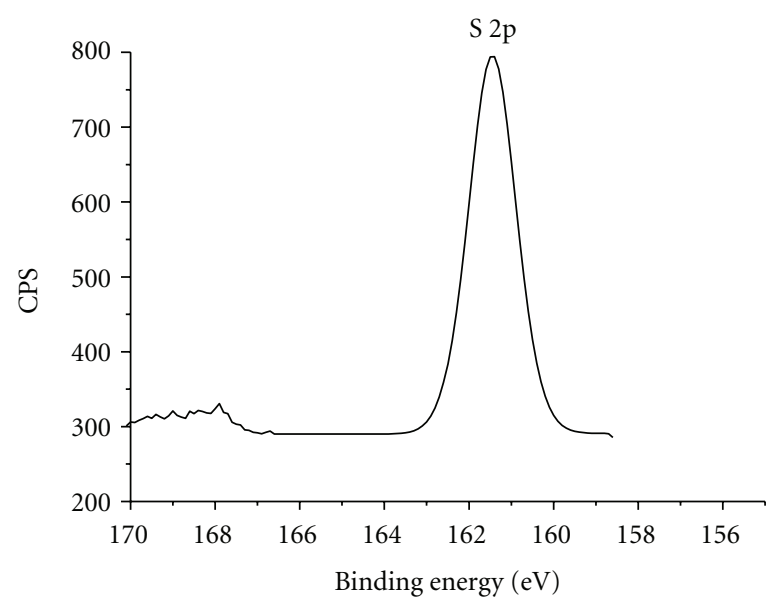

(d)

FIgURE 9: (a) XPS spectra of Cd, (b) Se, (c) Zn, and (d) S binding energy (eV) of CdSe-ZnS QDs, respectively.

shift is due to the further growth in the shell thickness. The initial broadening in PL is obviously from the growth of shell material, which is due to increase in particle size and further shell growth resulting in a red-shift (from $568 \mathrm{~nm}$ to $577 \mathrm{~nm}$ ) of PL [32].

Figure 7 is the HRTEM images of (a-b) CdSe and (cd) CdSe/ZnS core shell quantum dots. The lattice mismatch between the CdSe and $\mathrm{ZnS}$ is $12 \%$, and resolving this by using TEM is challenging $[28,32-34]$, but by measuring the average particle size on the HRTEM images of core, and by subtracting the average particle size measured on the HRTEM images of the core-shell will give an estimated shell thickness.

The average diameter of particle size measured on HRTEM image of CdSe/ZnS core-shell (Figure 7(b)) is $4.8 \mathrm{~nm}$ and the average diameter measured on the core (Figure 7(a)) is $3.6 \mathrm{~nm}$. Therefore, the average shell thickness on these particles is $1.2 \mathrm{~nm}$. Core-shell QDs were also characterized by energy dispersion spectroscopy (EDX) as show in Figure 7(e), and the EDX measurements were recorded in the scanning transmission electron microscopy (STEM) mode, which shows both core and shell material.
After ZnS shell growth, the XRD patterns of CdSe core were slightly shifted to higher 2-theta angle (Figure 8), which supports the formation of $\mathrm{ZnS}$ shell over the CdSe core. Further, broadening in XRD patterns represents the finite crystalline size of the core. It is interesting to note that in our previous study, when lower boiling point solvents (toluene $\left(\mathrm{BP} 110^{\circ} \mathrm{C}\right)$, $\mathrm{t}$-butyl toluene $\left(\mathrm{BP} 190^{\circ} \mathrm{C}\right)$ ) were used to digest as-prepared SMAD product, the particles retain the crystalline nature of the bulk starting material, that is, wurtzite (hexagonal) crystalline structure [1].

However, when digestive ripening was carried out at an elevated temperature $\left(250^{\circ} \mathrm{C}\right)$ in presence of phosphine and amine ligands, the particles attained a zinc blend (cubic) crystal structure. This transformation is most likely due to the reduction in growth rate [35] in presence of amine ligand [36]. Further, at elevated temperature, the crystalline defects are minimized resulting in enhanced quantum yield. Addition of inorganic high bang gap $\mathrm{ZnS}$ shell further enhanced the quantum yield from $36 \%$ to $60 \%$.

It is also important to note that when toluene was used as digestive ripening solvent, the measured QY was $11 \%$ and 


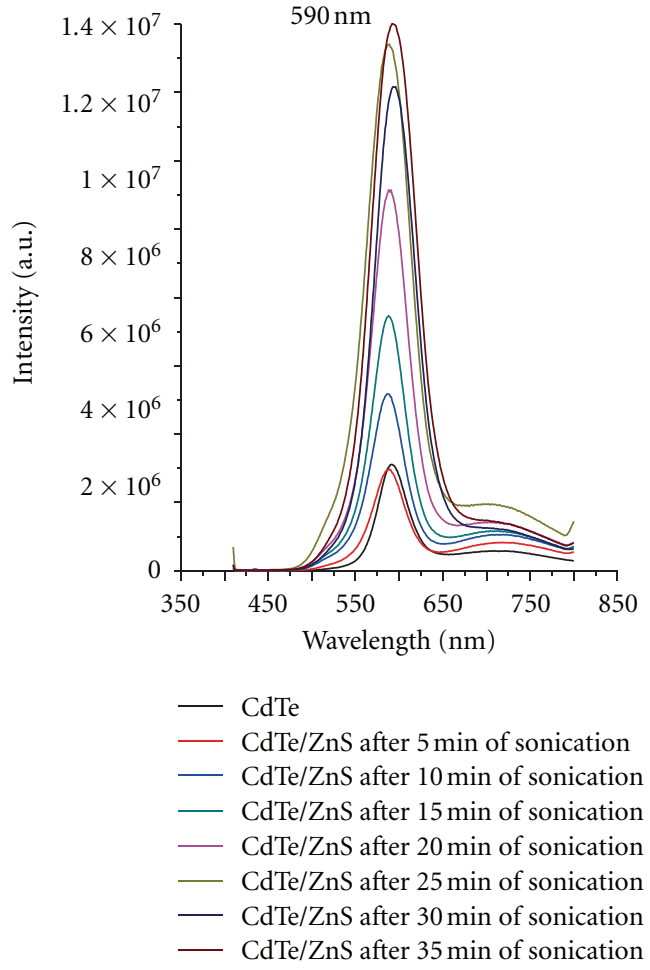

(a)

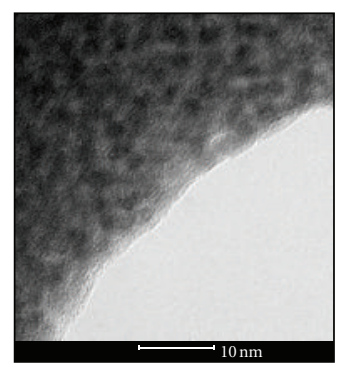

(b)

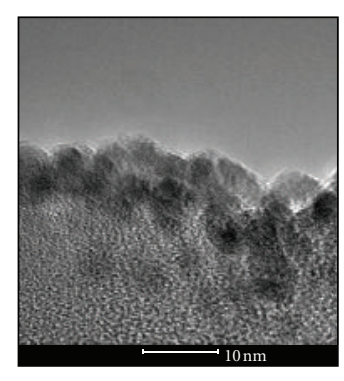

(c)

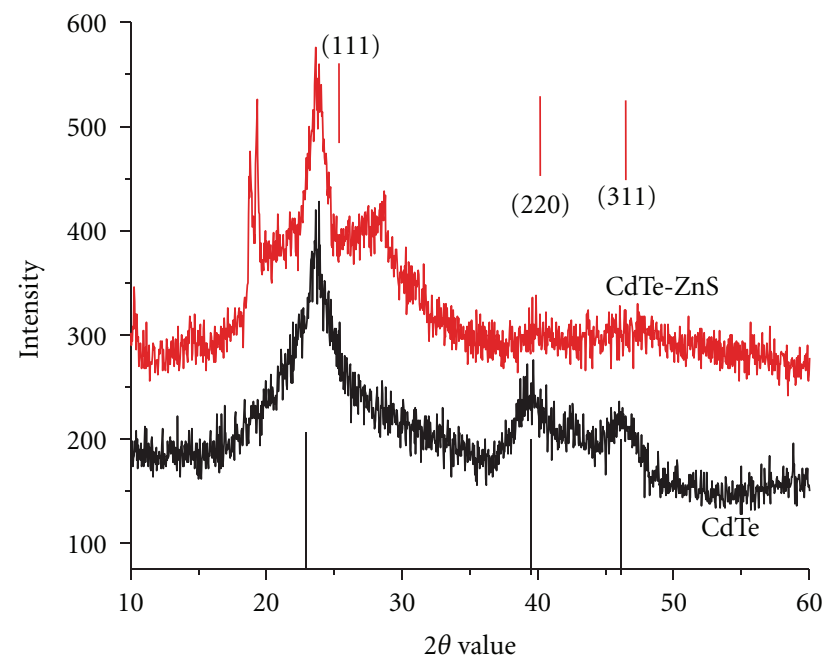

(e)

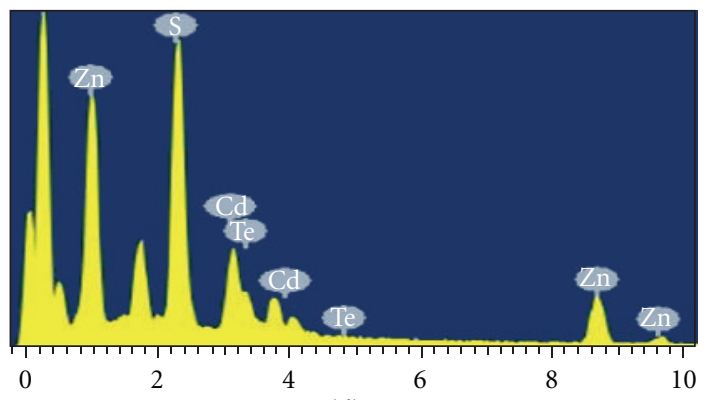

(d)

FIGURE 10: (a) Photoluminescence spectrum of CdTe/ZnS shell with sonication time, (b) HRTEM image of CdTeQDs after 30 min of digestive ripening, (c) after $\mathrm{ZnS}$ shell, (d) the characteristic zinc blend planes of 111,220 , and 311 locating at $24.40^{\circ}, 41.60^{\circ}$, and $47.90^{\circ}$ for CdTe core and at $24.94^{\circ}, 41.72^{\circ}$, and $48.76^{\circ}$ for $\mathrm{CdTe} / \mathrm{ZnS}$ in the $10-60^{\circ} 2 \varnothing$ range and (e) the energy dispersive X-ray spectroscopy (EDS) measurement showing the existence of cadmium, tellurium, zinc and sulfur.

a 28\% QY was achieved with t-butyltoluene [1]. With the higher digestive ripening temperature, the higher QY must be due to removal of crystalline defects. The HRTEM images (Figure 7) do show that the particles have good crystalline nature of both core and core-shell particles.

Surface composition of CdSe and CdSe/ZnS QDs was investigated with XPS. In Figure 9(a), the two strong peaks located at 405.2 and $412 \mathrm{eV}$ correspond to $\mathrm{Cd} 3 \mathrm{~d}$ binding energy of CdSe, and the peak at $54.3 \mathrm{eV}$ in Figure 9(b), corresponds to Se 3d binding energy of CdSe $[28,37]$. The XPS spectrum of CdSe/ZnS core/shell NCs shows typical peaks for $\mathrm{ZnS}$, with $\mathrm{Zn} 2 \mathrm{p}_{3 / 2}$ and $\mathrm{S} 2 \mathrm{p}_{3 / 2}$ located at 1022 (Figure 9(c)) and $161.5 \mathrm{eV}$ (Figure 9(d)), respectively. Based on XPS data, the growth of ZnS shell on CdSe core is confirmed.
4.4. Cadmium Telluride Core-Zinc Sulfide Shell QDs. Figure $10(\mathrm{a})$ is the PL of the $\mathrm{CdTe} / \mathrm{ZnS}$ core shell. The measured QY of CdTe and CdTe/ZnS is $38 \%$ and $60 \%$ respectively. The lattice parameters for $\mathrm{CdTe} \mathrm{c}=6.477 \AA$ and for $\mathrm{ZnS} c=6.257 \AA$ [38] and the lattice mismatch CdTe core and $\mathrm{ZnS}$ shell are $19.8 \%$ [38]. Figure 10(b) and 10(c) are the HRTEM image of CdTe QDs before and after addition of $\mathrm{ZnS}$ shell. In order to confirm the formation of $\mathrm{ZnS}$ shell on CdTe, energy dispersive X-ray spectroscopy (EDX) and the powder XRD measurements were made. The EDX spectrum in Figure 10(d) shows the existence of Te, Cd, Zn, and $\mathrm{S}$ in the $\mathrm{CdTe} / \mathrm{ZnS}$ sample. The sulfur peak at $2.3 \mathrm{keV}$ and the $\mathrm{Zn}$ peak at $8.9 \mathrm{keV}$ in EDX spectrum indicate the existence of a $\mathrm{ZnS}$ shell layer on the CdTe core. These results of the EDX spectrum further confirm the formation of the core/shell QDs. 


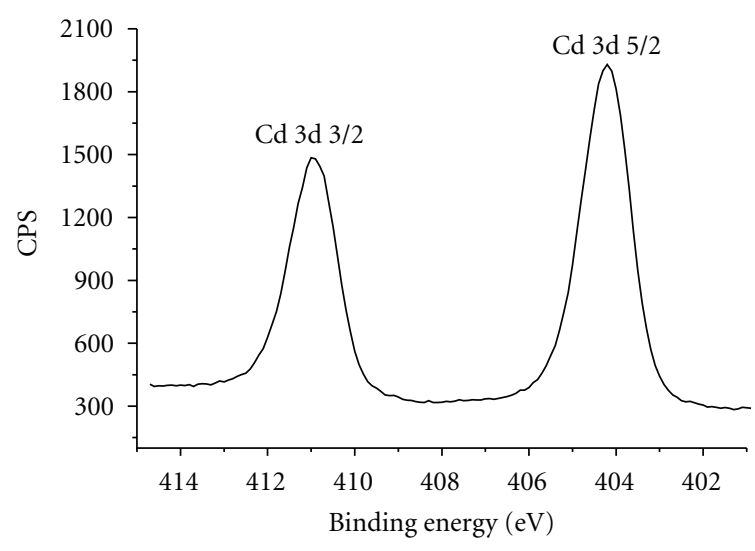

(a)

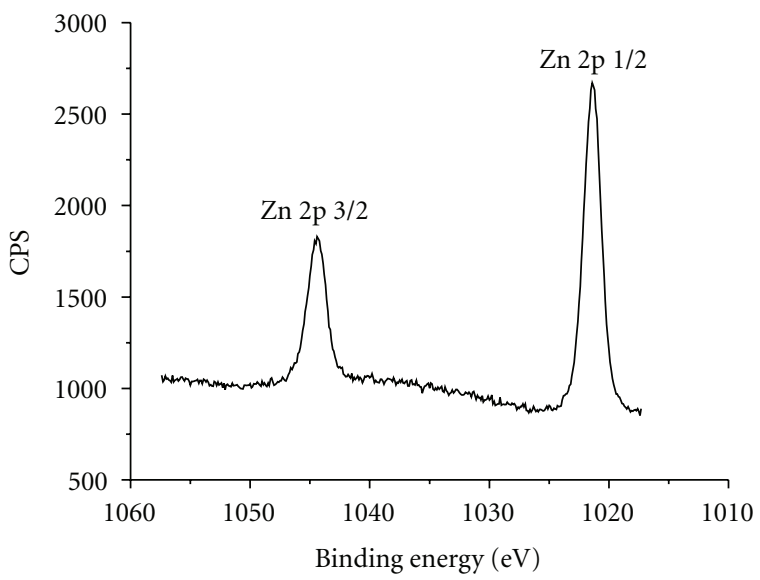

(c)

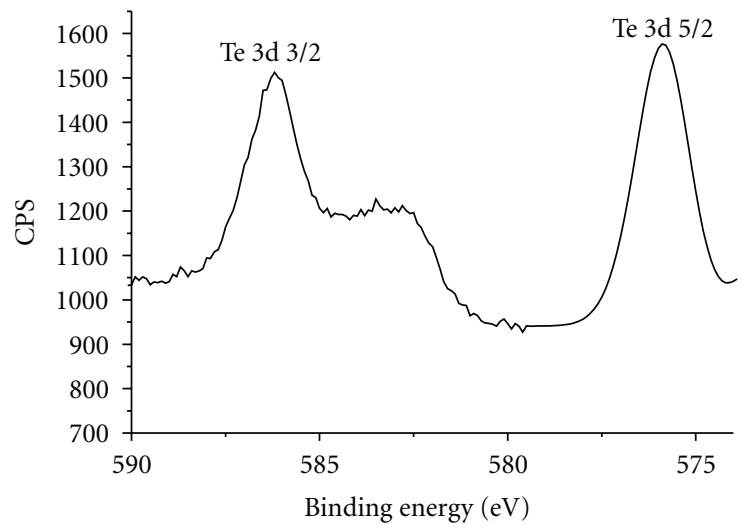

(b)

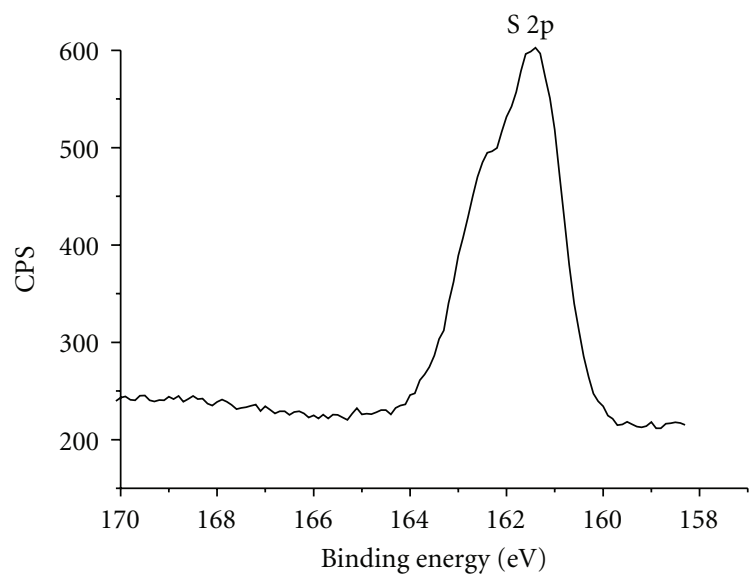

(d)

FIgUre 11: (a) XPS spectra of Cd, (b) Te, (c) Zn, and, (d) S-binding energy (eV) of CdTe-ZnS QDs, respectively.

The powder XRD patterns of CdTe and CdTe/ZnS core shell are shown in Figure 10(e). The characteristic zinc blend planes of 111,220 , and 311 located at $24.40^{\circ}, 41.60^{\circ}$, and $47.90^{\circ}$ for CdTe core and at $24.94^{\circ}, 41.72^{\circ}$, and $48.76^{\circ}$ for $\mathrm{CdTe} / \mathrm{ZnS}$ in $10-60^{\circ} 2 \varnothing$ range are observed. The position of the XRD peaks of CdTe cores matched well with those of bulk CdTe cubic structure (JCPDS No. 15-0770). After growth of $\mathrm{ZnS}$ shell on the CdTe core, the peak position shifted to higher angles towards the positions of bulk $\mathrm{ZnS}$ cubic structure peaks (JCPDS No. 05-0566), which substantiates the formation of $\mathrm{CdTe} / \mathrm{ZnS}$ core shell.

In Figure 11(a), the two strong peaks located at 404.9 and $412 \mathrm{eV}$ correspond to Cd 3d-binding energy of CdTe QD, and the peak at $571.8,581.9 \mathrm{eV}$ in Figure 11(b) corresponds to $\mathrm{Te} 3 \mathrm{~d}$ binding energy of CdTe. In Figure 11(c), the strong peaks $1021,1044 . \mathrm{eV}$ correspond to $\mathrm{Zn} 2 \mathrm{p}$ and $161.5 \mathrm{eV}$ in Figure 11(d), correspond to S binding energy of CdTeZnS QDs. These data of XPS provide the direct evidence of the formation of CdTe/ZnS QDs.

\section{Conclusions}

We demonstrated the ability of the SMAD technique for the synthesis of semiconductor cadmium selenide and cadmium telluride quantum dots. Digestive ripening at hightemperature $\left(250^{\circ} \mathrm{C}\right)$ was achieved by employing TOPO and $\mathrm{OA}$ as digestive ripening solvents. Compared to CdSe (90 $\mathrm{min}$ ), the digestive ripening of CdTe was much faster (60 $\mathrm{min}$ ). High temperature digestive ripening facilitated the removal of crystalline defects and allows enhancement in the photoluminescence of both CdSe and CdTe. Further enhancement of QY (40 to 60\%) was achieved by coating with a high band gap inorganic $\mathrm{ZnS}$ shell by sonochemical procedure. Formation of $\mathrm{ZnS}$ over the core is evident from enhanced PL, XRD, EDX, and XPS analysis.

\section{Acknowledgment}

The support of Department of Energy (DE-FG 02-10 ER16202) is gratefully acknowledged.

\section{References}

[1] S. Cingarapu, Z. Yang, C. M. Sorensen, and K. J. Klabunde, "Synthesis of CdSe quantum dots by evaporation of bulk CdSe using SMAD and digestive ripening processes," Chemistry of Materials, vol. 21, no. 7, pp. 1248-1252, 2009.

[2] S. Cingarapu, Z. Yang, C. M. Sorensen, and K. J. Klabunde, "Synthesis of indium nanoparticles: digestive ripening under 
mild conditions," Inorganic Chemistry, vol. 50, no. 11, pp. 5000-5005, 2011.

[3] D. Heroux, A. Ponce, S. Cingarapu, and K. J. Klabunde, "Nanoparticles prepared by salt vapor-solvent vapor cocondensation and controlled nucleation: metal sulfides (ZnS, CdS, $\mathrm{CdSe}, \mathrm{PbS}$ ), and metal halide (LiF). Size, aggregates, structures, digestive ripening, superlattices, and impregnations," Advanced Functional Materials, vol. 17, no. 17, pp. 3562-3568, 2007.

[4] A. A. Ponce and K. J. Klabunde, "Chemical and catalytic activity of copper nanoparticles prepared via metal vapor synthesis," Journal of Molecular Catalysis A, vol. 225, no. 1, pp. 1-6, 2005.

[5] A. B. Smetana, K. J. Klabunde, and C. M. Sorensen, "Synthesis of spherical silver nanoparticles by digestive ripening, stabilization with various agents, and their 3-D and 2-D superlattice formation," Journal of Colloid and Interface Science, vol. 284, no. 2, pp. 521-526, 2005.

[6] S. Stoeva, K. J. Klabunde, C. M. Sorensen, and I. Dragieva, "Gram-scale synthesis of monodisperse gold colloids by the solvated metal atom dispersion method and digestive ripening and their organization into two- and three-dimensional structures," Journal of the American Chemical Society, vol. 124, no. 10, pp. 2305-2311, 2002.

[7] S. I. Stoeva, A. B. Smetana, C. M. Sorensen, and K. J. Klabunde, "Gram-scale synthesis of aqueous gold colloids stabilized by various ligands," Journal of Colloid and Interface Science, vol. 309, no. 1, pp. 94-98, 2007.

[8] S. B. Kalidindi and B. R. Jagirdar, "Highly monodisperse colloidal magnesium nanoparticles by room temperature digestive ripening," Inorganic Chemistry, vol. 48, no. 10, pp. 45244529, 2009.

[9] X. M. Lin, C. M. Sorensen, and K. J. Klabunde, "Digestive ripening, nanophase segregation and superlattice formation in gold nanocrystal colloids," Journal of Nanoparticle Research, vol. 2, no. 2, pp. 157-164, 2000.

[10] K. J. Klabunde, C. M. Sorensen, S. I. Stoeva, B. L. V. Prasad, A. B. Smetana, and X. M. Lin, "Digestive ripening, or "nanomachining," to achieve nanocrystal size control," in Metal Clusters in Catalysis and Materials Science: The Issue of Size Control, Part II Methodologies, C. Corrain, G. Schmid, and N. Toshima, Eds., chapter 11, pp. 233-252, Elsevier Science, Amsterdam, Holland, 2008.

[11] D. S. Sidhaye and B. L. V. Prasad, "Many manifestations of digestive ripening: monodispersity, superlattices and nanomachining," New Journal of Chemistry, vol. 35, no. 4, pp. 755-763, 2011.

[12] C. B. Murray, D. J. Norris, and M. G. Bawendi, "Synthesis and characterization of nearly monodisperse CdE (E = sulfur, selenium, tellurium) semiconductor nanocrystallites," Journal of the American Chemical Society, vol. 115, no. 19, pp. 8706$8715,1993$.

[13] D. Battaglia, J. J. Li, Y. Wang, and X. Peng, "Colloidal twodimensional systems: CdSe quantum shells and wells," Angewandte Chemie-International Edition, vol. 42, no. 41, pp. 5035-5039, 2003.

[14] X. Peng, L. Manna, W. Yang et al., "Shape control of CdSe nanocrystals," Nature, vol. 404, no. 6773, pp. 59-61, 2000.

[15] Z. A. Peng and X. Peng, "Formation of high-quality CdTe, CdSe, and CdS nanocrystals using $\mathrm{CdO}$ as precursor," Journal of the American Chemical Society, vol. 123, no. 1, pp. 183-184, 2001.
[16] L. Qu, Z. A. Peng, and X. Peng, "Alternative routes toward high quality CdSe nanocrystals," Nano Letters, vol. 1, no. 6, pp. 333337, 2001.

[17] D. V. Talapin, A. L. Rogach, A. Kornowski, M. Haase, and H. Weller, "Highly luminescent monodisperse CdSe and $\mathrm{CdSe} / \mathrm{ZnS}$ nanocrystals synthesized in a hexadecylamine-trioctylphosphine oxide-trioctylphospine mixture," Nano Letters, vol. 1, no. 4, pp. 207-211, 2001.

[18] H. Yang, N. Fan, W. Luan, and S. T. Tu, "Synthesis of monodisperse nanocrystals via microreaction: open-to-air synthesis with oleylamine as a coligand," Nanoscale Research Letters, vol. 4, no. 4, pp. 344-352, 2009.

[19] W. W. Yu and X. Peng, "Formation of high-quality CdS and other II-VI semiconductor nanocrystals in noncoordinating solvents: tunable reactivity of monomers," Angewandte Chemie-International Edition, vol. 41, no. 13, pp. 2368-2371, 2002.

[20] M. A. Hines and P. Guyot-Sionnest, "Synthesis and characterization of strongly luminescing ZnS-capped CdSe nanocrystals," Journal of Physical Chemistry, vol. 100, no. 2, pp. 468471, 1996.

[21] D. V. Talapin, I. Mekis, S. Gotzinger, A. Kornowski, O. Benson, and $\mathrm{H}$. Weller, "CdSe/CdS/ZnS and CdSe/ZnSe/ZnS coreshell-shell nanocrystals," Journal of Physical Chemistry B, vol. 108, no. 49, pp. 18826-18831, 2004.

[22] B. R. Dorvel, H. M. Keizer, D. Fine, J. Vuorinen, A. Dodabalapur, and R. S. Duran, "Formation of tethered bilayer lipid membranes on gold surfaces: QCM-Z and AFM study," Langmuir, vol. 23, no. 13, pp. 7344-7355, 2007.

[23] X. S. Wang, T. E. Dykstra, M. R. Salvador, I. Manners, G. D. Scholes, and M. A. Winnik, "Surface passivation of luminescent colloidal quantum dots with poly(dimethylaminoethly methacrylate) through a ligand exchange process," Journal of the American Chemical Society, vol. 126, no. 25, pp. 7784-7785, 2004.

[24] M. J. Murcia, D. L. Shaw, H. Woodruff, C. A. Naumann, B. A. Young, and E. C. Long, "Facile sonochemical synthesis of highly luminescent ZnS-shelled CdSe quantum dots," Chemistry of Materials, vol. 18, no. 9, pp. 2219-2225, 2006.

[25] J. K. Klabunde, P. L. Timms, P. S. Skell, and S. Ittel, "Introduction to metal atom syntheses," in Inorganic Syntheses, D. Shriver, Ed., vol. 19, pp. 59-86, John Wiley \& Sons, New York, NY, USA, 1979.

[26] P. S. Nair, T. Radhakrishnan, N. Revaprasadu, G. Kolawole, and P. O'Brien, "Cadmium ethylxanthate: a novel single-source precursor for the preparation of CdS nanoparticles," Journal of Materials Chemistry, vol. 12, no. 9, pp. 2722-2725, 2002.

[27] S. L. Cumberland, K. M. Hanif, A. Javier et al., "Inorganic clusters as single-source precursors for preparation of CdSe, ZnSe, and CdSe/ZnS nanomaterials," Chemistry of Materials, vol. 14, no. 4, pp. 1576-1584, 2002.

[28] C. Q. Zhu, P. Wang, X. Wang, and Y. Li, "Facile phosphine-free synthesis of CdSe/ZnS core/shell nanocrystals without precursor injection," Nanoscale Research Letters, vol. 3, no. 6, pp. 213-220, 2008.

[29] X. Zhong, Y. Feng, and Y. Zhang, "Facile and reproducible synthesis of red-emitting CdSe nanocrystals in amine with longterm fixation of particle size and size distribution," The Journal of Physical Chemistry C, vol. 111, no. 2, pp. 526-531, 2006.

[30] Y. M. Sung, K. S. Park, Y. J. Lee, and T. G. Kim, "Ripening kinetics of CdSe/ZnSe core/shell nanocrystals," The Journal of Physical Chemistry C, vol. 111, no. 3, pp. 1239-1242, 2007. 
[31] D. S. Sidhaye and B. L. V. Prasad, "Many manifestations of digestive ripening: monodispersity, superlattices and nanomachining," New Journal of Chemistry, vol. 124, no. 9, pp. 2049 2055, 2011.

[32] L. Qu and X. Peng, "Control of photoluminescence properties of CdSe nanocrystals in growth," Journal of the American Chemical Society, vol. 124, no. 9, pp. 2049-2055, 2002.

[33] B. O. Dabbousi, J. Rodriguez-Viejo, F. V. Mikulec et al., "(CdSe) ZnS core-shell quantum dots: synthesis and characterization of a size series of highly luminescent nanocrystallites," The Journal of Physical Chemistry B, vol. 101, no. 46, pp. 94639475, 1997.

[34] M. A. Hines and P. Guyot-Sionnest, "Bright UV-blue luminescent colloidal ZnSe nanocrystals," The Journal of Physical Chemistry B, vol. 102, no. 19, pp. 3655-3657, 1998.

[35] M. B. Mohamed, D. Tonti, A. Al-Salman, A. Chemseddine, and M. Chergui, "Synthesis of high quality zinc blende CdSe nanocrystals," The Journal of Physical Chemistry B, vol. 109, no. 21, pp. 10533-10537, 2005.

[36] L. Wang, X. Sun, W. Liu, and X. Yu, "Phosphine-free synthesis of high quality zinc-blende CdSe nanocrystals in air," Colloids and Surfaces A, vol. 353, no. 2-3, pp. 232-237, 2010.

[37] Z. Quan, Z. Wang, P. Yang, J. Lin, and J. Fang, "Synthesis and characterization of high-quality $\mathrm{ZnS}, \mathrm{ZnS}: \mathrm{Mn}^{2+}$, and $\mathrm{ZnS}: \mathrm{Mn}^{2+} / \mathrm{ZnS}$ (core/shell) luminescent nanocrystals," Inorganic Chemistry, vol. 46, no. 4, pp. 1354-1360, 2007.

[38] T. Trindade, P. O’Brien, and N. L. Pickett, "Nanocrystalline semiconductors: synthesis, properties, and perspectives," Chemistry of Materials, vol. 13, no. 11, pp. 3843-3858, 2001. 

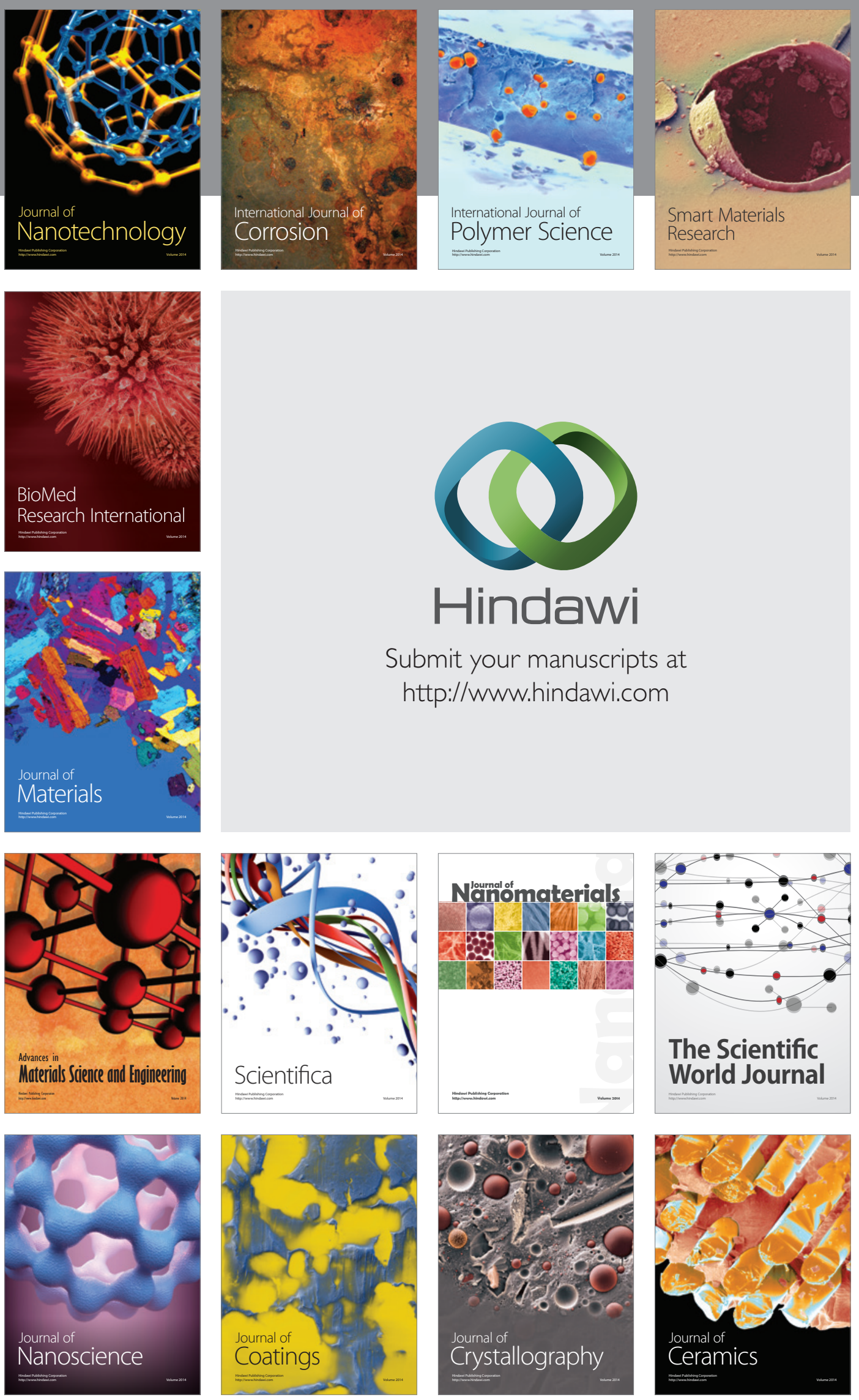

The Scientific World Journal

Submit your manuscripts at

http://www.hindawi.com

\section{World Journal}

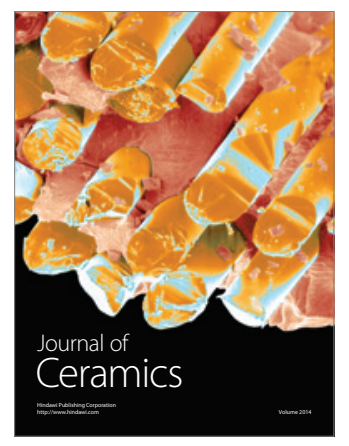

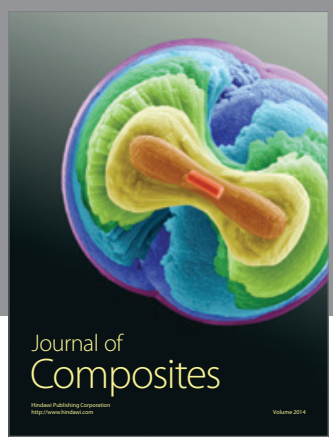
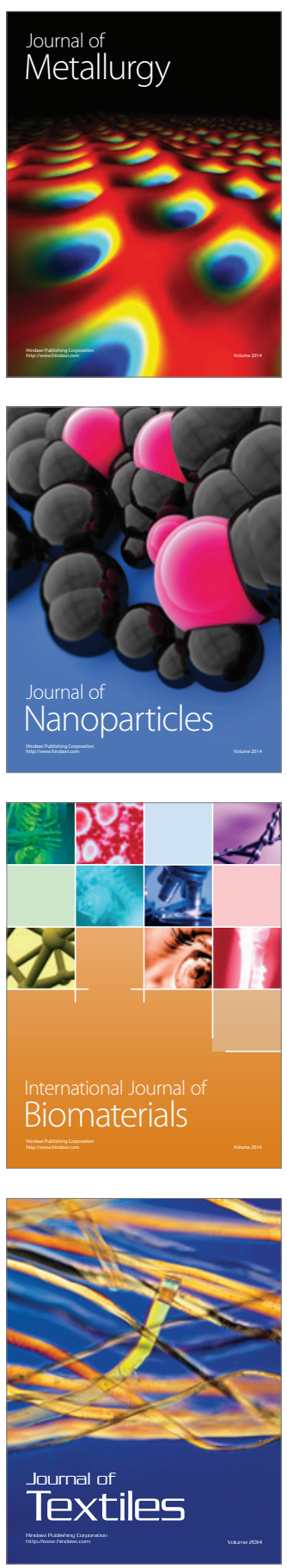